\title{
IUCrJ
}

Volume 7 (2020)

Supporting information for article:

Charge density view on bicalutamide molecular interactions in the monoclinic polymorph and androgen receptor binding pocket

Alexander A. Korlyukov, Maura Malinska, Anna V. Vologzhanina, Mikhail S. Goizman, Damian Trzybinski and Krzysztof Wozniak 


\title{
Supporting Information
}

\section{Charge density view on bicalutamide molecular interactions in monoclinic polymorph and androgen receptor binding pocket}

\author{
Alexander A. Korlyukov ${ }^{1 * *}$, Maura Malinska ${ }^{2 \ddagger}$, Anna V. Vologzhanina1, Mikhail S. \\ Goizman $^{3}$, Damian Trzybinski ${ }^{2}$ and Krzysztof Wozniak ${ }^{2 *}$ \\ ${ }^{1}$ A.N.Nesmeyanov Institute of Organoelement Compounds of Russian Academy of Sciences, \\ 119991, Vavilov St. 28, Moscow, Russian Federation \\ ${ }^{3}$ Biological and Chemical Research Centre, Department of Chemistry, University of Warsaw, \\ Żwirki i Wigury 101, 02089 Warszawa, Poland \\ ${ }^{2}$ Drug Technology Co, 2a Rabochaya St, Chimki 141400, Moscow Oblast, Russia
}

Corresponding addresses: akorlyukov@gmail.com and kwozniak@chem.uw.edu.pl

Multipole refinement of Bic

\section{Contents}

Residual electron density distribution 3

Fractal plot

Normal probability plot

Critical points

Experimental deformation density

Energy framework for crystal packing

Characteristics of intramolecular CPs

The QTAIM integrated parameters

Parameters of intermolecular contacts

Bicalutamide in the binding pocket

Electrostatic interaction energy

References

2

4

5

6

7

8

9

11

12

13

14

15 


\section{Multipole refinement of Bic.}

The model neglecting anharmonic motion for the $S(1)$ atom and the $\mathrm{O}(4)$ atom of the carbonyl group (Fig. S1, left) shows the shashlik-like pattern of residual density which is typical for unmodeled anharmonic motion [S1]. It disappears after inclusion of anharmonic nuclear motion in the form of the Gram-Charlier tensors of the third order for the oxygen atom and of the fourth order for the S(1) atom (Fig. S1, right). In order to avoid an abundant parameters refinement only the non-zero Gram-Charlier parameters were refined, while the values of the Gram-Charlier parameters equal to zero within three e.s.d. were set to zero for further refinement. Non-zero values were obtained for seven parameters for the $\mathrm{O}(4)$ atom $\left(\mathrm{C}^{111}, \mathrm{C}^{333}, \mathrm{C}^{112}, \mathrm{C}^{113}, \mathrm{C}^{133}, \mathrm{C}^{233}, \mathrm{C}^{123}\right)$ and three parameters for the $\mathrm{S}(1)$ atom $\left(\mathrm{C}^{222}, \mathrm{C}^{333}, \mathrm{D}^{2222}\right)$. These Gram-Charlier parameters were refined with the positional and thermal parameters keeping all multipolar parameters fixed. In the next step, only the multipolar parameters were refined. In all further steps of the refinement the above mentioned Gram-Charlier coefficients were fixed at the obtained values and the other coefficients were set to zero.
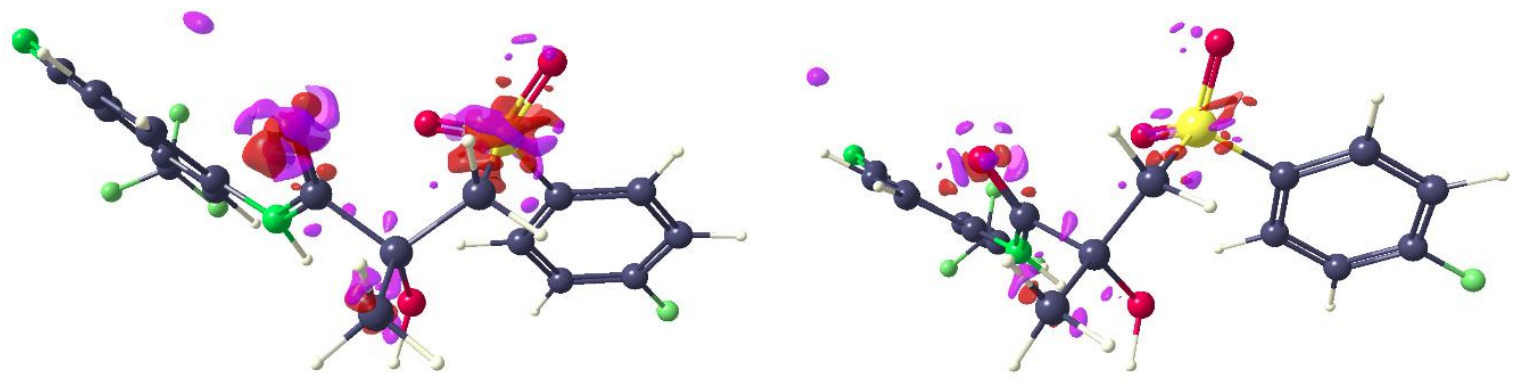

Figure S1 Arrangement of molecules in crystal structure of Bic and the isosurface representation (contour value $=0.20 \mathrm{e} \AA^{-3}$; red is positive, fuchsia is negative) of the residual density in various refinement models of Bic: (left) a model neglecting anharmonic nuclear motion; (right) a model including anharmonic nuclear motion to third order for the $\mathrm{O}(4)$ atoms and to fourth order for the S(1) atom. 


\section{Residual density distribution.}

The residual density maps (Figure S2) for Bic demonstrate that there still remains some small unfitted electron density at $\mathrm{S}(1)$ and $\mathrm{O}(4)$ atoms that can be accounted for unresolved anharmonic motion. Inspection of the residual density with jnk2RDA developed by Meindl \& Henn [S2] results in the distribution shown in Figures S4 and S5. The deviation from the ideal Gaussian shape for 1 originates mainly from the not ideal description of above mentioned atoms. $\mathrm{d}^{\mathrm{f}}(0)=2.71$ is similar with values given in paper describing this procedure suggesting a successful refinement. Broadening of the distribution results in relatively high $e_{\text {gross }}$ value and may be accounted for insufficiently accurate s.u. values of intensities measured with CCD area detector. Normal probability plot plotted against full dataset and time scale factor plot vs. the resolution confirms good data quality over the whole resolution range and its internal consistency (Fig. S6) of 1.
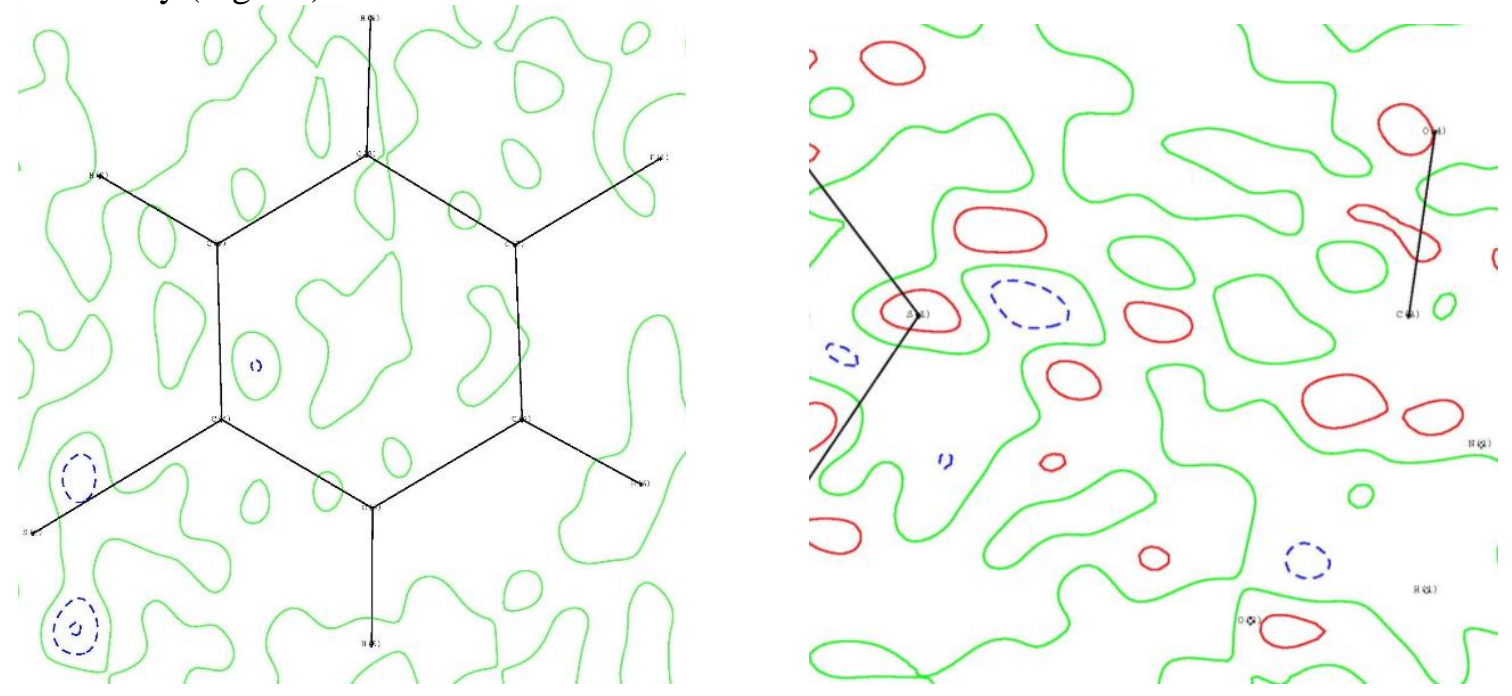

Figure S2 The residual density maps in the sections of (left) $\mathrm{C}(4), \mathrm{C}(6)$ and $\mathrm{C}(8)$; (right) $\mathrm{S}(1), \mathrm{C}(3)$ and $\mathrm{O}(4)$ atoms calculated using all data for Bic. Isocontours are drawn every 0.1 e $\AA^{-3}$; positive contours are shown in red, the negative contours are dashed blue. 
fractal dimension $\left(d^{f}\right)$ vs. residual density $\left(\rho_{0}\right)$

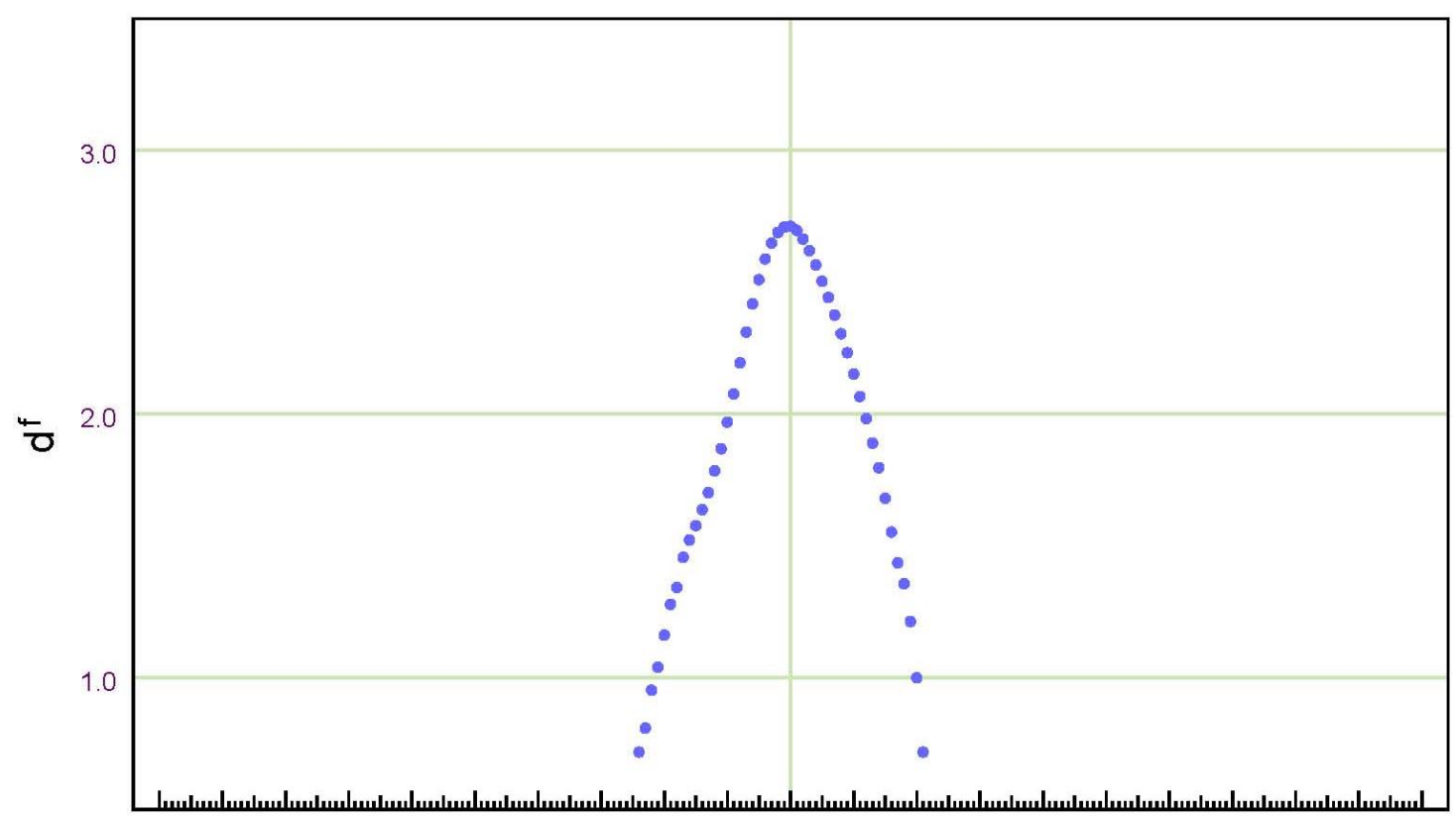

$\begin{array}{lllllllllllllllllllll}-1.0 & -0.9 & -0.8 & -0.7 & -0.6 & -0.5 & -0.4 & -0.3 & -0.2 & -0.1 & 0.0 & 0.1 & 0.2 & 0.3 & 0.4 & 0.5 & 0.6 & 0.7 & 0.8 & 0.9 & 1.0\end{array}$

\section{$\rho_{0}\left[e \AA^{-3}\right]$}

MODEL *model 4400

FOUR fmod14400 fmod2-1200

SELECT *fobs *fmod1 fmod2 print snlmin 0. snlmax 2.

GRID 3-points perp *cryst

ATOM label S(1) symm 1 trans $0000^{*}$ mark on plot

ATOM label $\mathrm{C}(3)$ symm 1 trans 000 *mark on plot

ATOM label $O(4)$ symm 1 trans 000 "mark on plot

LIMITS $x \min 0.0 \times \max 1.0 \mathrm{nx} 70$

IMUISS ymin 00 Vmax $10 n y 60$ $d^{\dagger}(0)=2.7118$

$\rho_{\min }(d=2)=-0.0971$ eA^-3

$\rho_{\max }(\mathrm{d}=2)=0.1180 \mathrm{eA}-3$

$\mathrm{nx}=70$ rho_min: -0.24 eA^-3

ny $=60$ rho_max: 0.22 eA^-3

$\mathrm{nz}=50$ delta rho: $0.46 \mathrm{eA}^{\wedge}-3$

Figure S3 Plot of the fractal dimension $\mathrm{d}^{\mathrm{f}} \mathrm{vs}$. the residual electron density $\left(\rho_{0}\right)$ in the unit cell of Bic.

No resolution cutoff was applied to the data used for the Fourier transformation. 


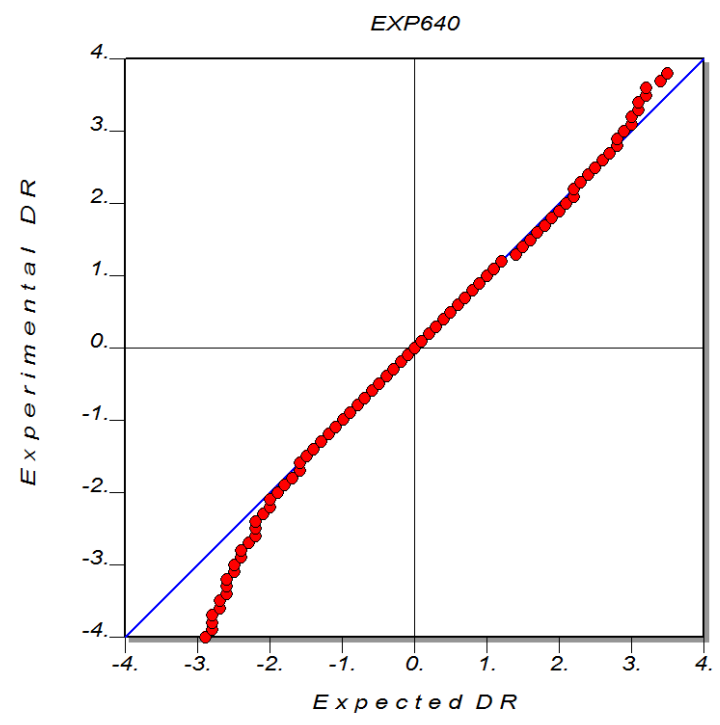

(a)

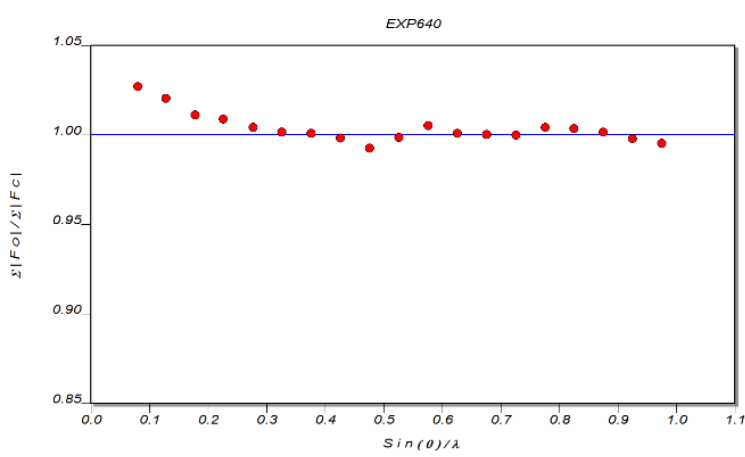

(b)

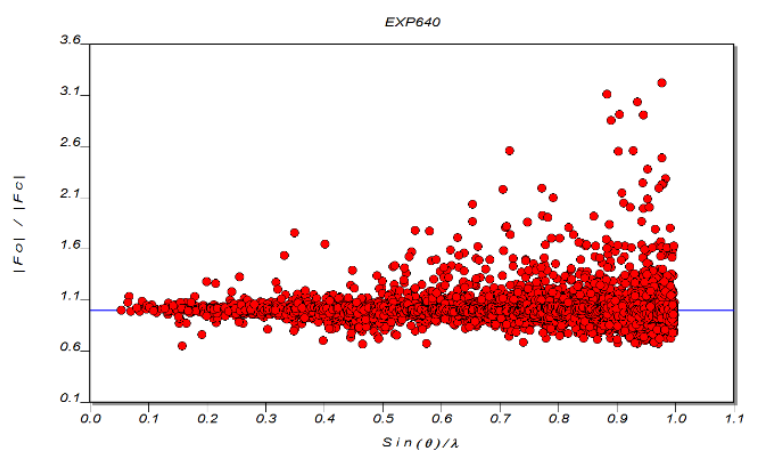

(c)

Figure S4 (a) Normal probability plot plotted against full dataset of Bic; (b) Scale factor plot against resolution; (c) $\left|\mathrm{F}_{\mathrm{o}}\right| /\left|\mathrm{F}_{\mathrm{c}}\right|$ against resolution. 


\section{Charge Density Properties}

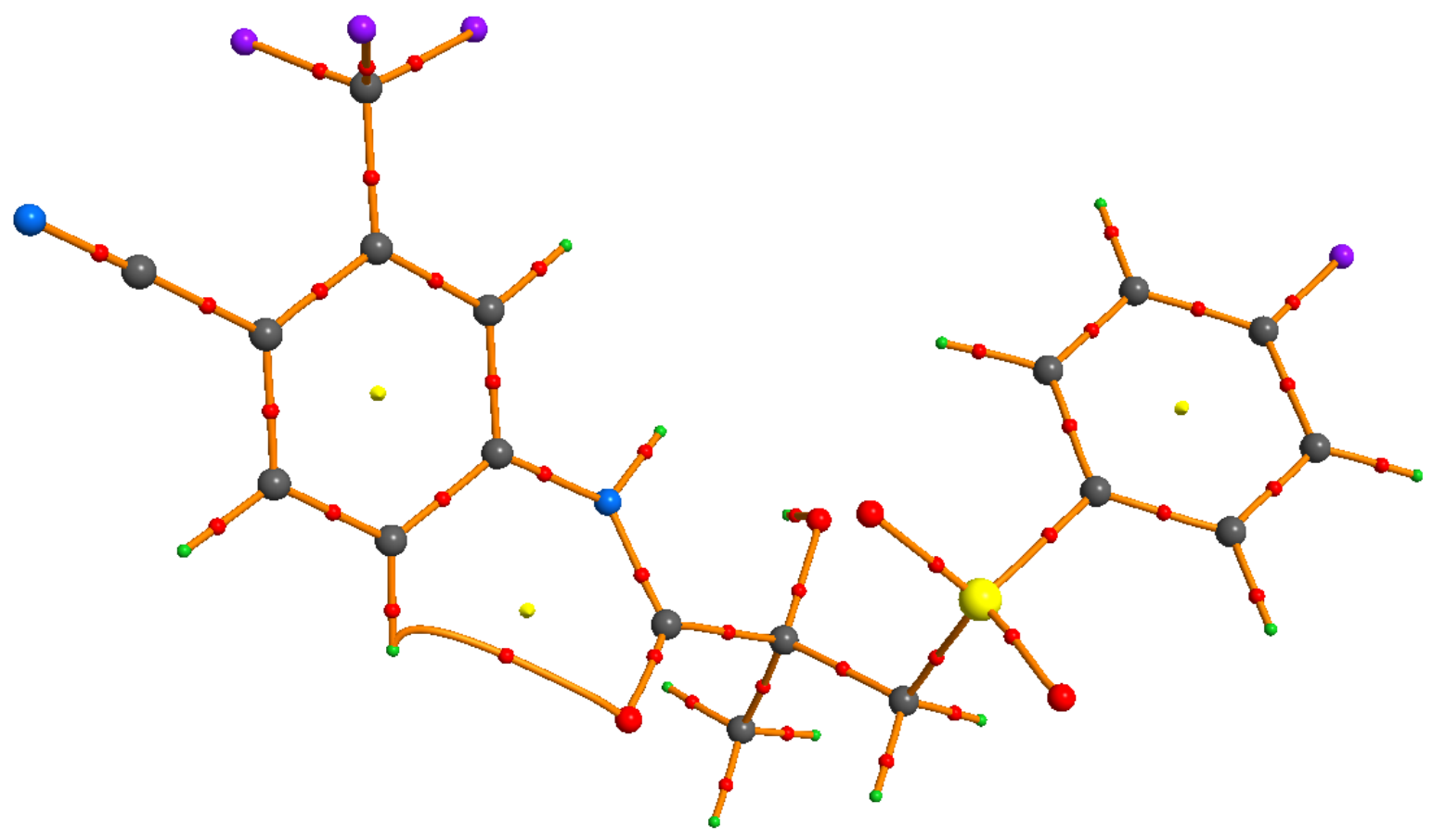

Figure S5 Molecular graph of Bic. Red and yellow circles denote CP $(3,-1)$ and $(3,+1)$.

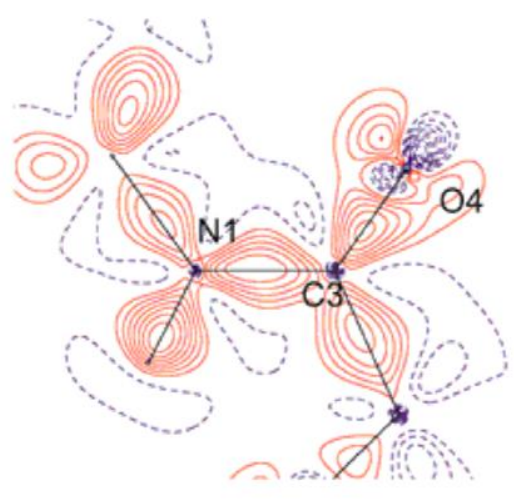

(a)

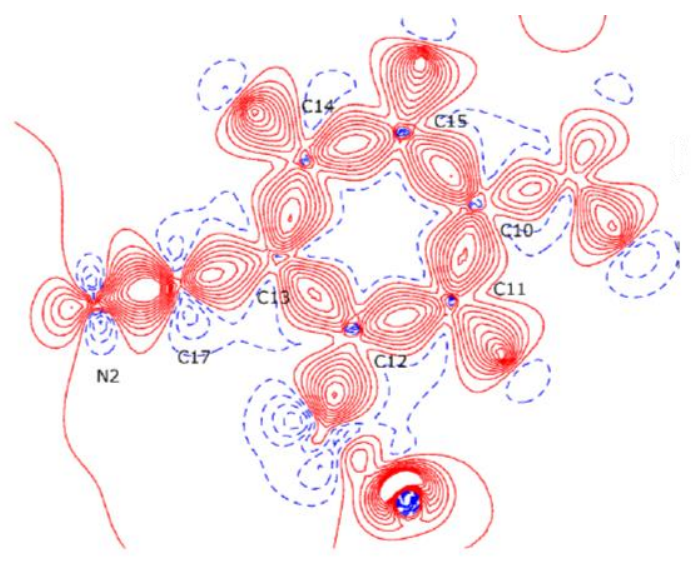

(b)

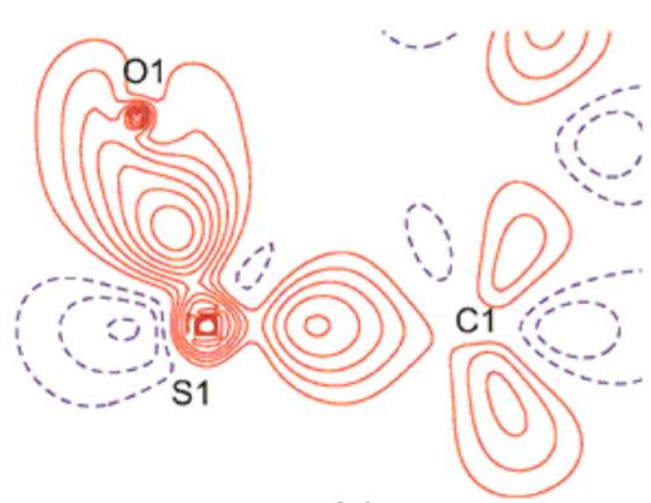

(c)

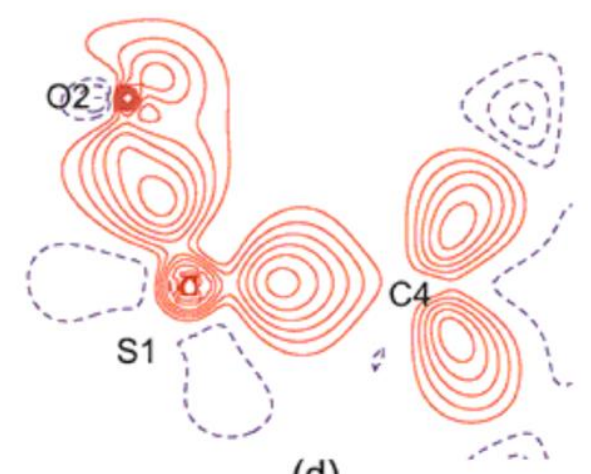

(d)

Figure S6 Experimental static deformation density in Bic depicted in the sections of (a) N(1), C(3), $\mathrm{O}(4)$; (b) $\mathrm{N}(2), \mathrm{C}(11), \mathrm{C}(15)$; (c) $\mathrm{S}(1), \mathrm{O}(1), \mathrm{C}(1)$; (d) $\mathrm{S}(1) \mathrm{O}(2), \mathrm{C}(4)$ atoms. The contour levels are equal to $\pm 0.1, \pm 0.2, \pm 0.3, \pm 0.4, \pm 0.5, \pm 0.6, \pm 0.7, \pm 0.8, \pm 0.9, \pm 1.0$ e $\AA^{-3}$; the positive contours are shown with solid red line, the negative contours are dashed blue. 


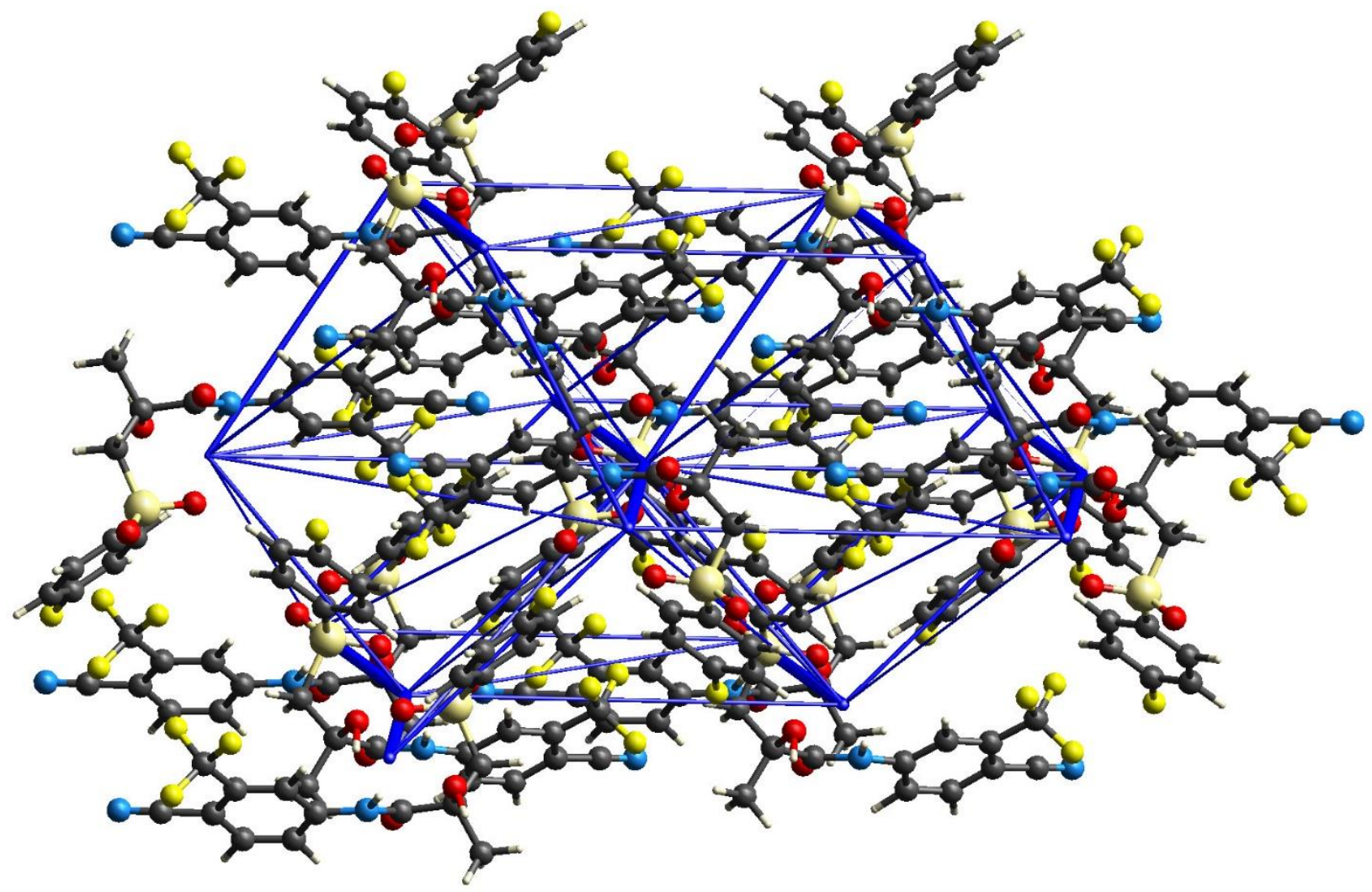

Figure S7 Energy framework for crystal packing of Bic. The width of blue line is related to the strength of intermolecular binding $\left(\mathrm{E}_{\mathrm{tot}}\right)$. 
Table S1 Characteristics of intramolecular CPs of Bic [a.u.]

\begin{tabular}{|c|c|c|c|c|c|c|}
\hline Type CP & Bond & $\rho(r)$ & $\nabla^{2} \rho(\mathrm{r})$ & $g(r)$ & $v(r)$ & $h(r)$ \\
\hline$(3,-1)$ & $\mathrm{S}(1)-\mathrm{O}(1)$ & 0.382 & -0.313 & 0.524 & -1.127 & -0.602 \\
\hline$(3,-1)$ & $\mathrm{S}(1)-\mathrm{O}(2)$ & 0.359 & -0.314 & 0.469 & -1.016 & -0.547 \\
\hline$(3,-1)$ & $S(1)-C(1)$ & 0.212 & -0.292 & 0.167 & -0.407 & -0.240 \\
\hline$(3,-1)$ & $S(1)-C(4)$ & 0.231 & -0.453 & 0.175 & -0.463 & -0.288 \\
\hline$(3,-1)$ & $\mathrm{F}(1)-\mathrm{C}(8)$ & 0.283 & -0.737 & 0.227 & -0.638 & -0.411 \\
\hline$(3,-1)$ & $\mathrm{F}(2)-\mathrm{C}(16)$ & 0.310 & -0.676 & 0.296 & -0.760 & -0.465 \\
\hline$(3,-1)$ & $\mathrm{F}(3)-\mathrm{C}(16)$ & 0.334 & -1.767 & 0.167 & -0.776 & -0.609 \\
\hline$(3,-1)$ & $\mathrm{F}(4)-\mathrm{C}(16)$ & 0.320 & -1.784 & 0.132 & -0.711 & -0.578 \\
\hline$(3,-1)$ & $\mathrm{O}(3)-\mathrm{C}(18)$ & 0.291 & -1.022 & 0.196 & -0.647 & -0.451 \\
\hline$(3,+1)$ & & 0.022 & 0.138 & 0.028 & -0.021 & 0.007 \\
\hline$(3,-1)$ & $\mathrm{O}(3)-\mathrm{H}(3)$ & 0.325 & -1.227 & 0.238 & -0.782 & -0.544 \\
\hline$(3,-1)$ & $\mathrm{O}(3)-\mathrm{H}(1)$ & 0.025 & 0.106 & 0.024 & -0.021 & 0.003 \\
\hline$(3,-1)$ & $\mathrm{O}(4)-\mathrm{C}(3)$ & 0.422 & -1.384 & 0.452 & -1.250 & -0.798 \\
\hline$(3,-1)$ & $\mathrm{O}(4)-\mathrm{H}(15)$ & 0.015 & 0.058 & 0.012 & -0.010 & 0.002 \\
\hline$(3,-1)$ & $\mathrm{N}(1)-\mathrm{C}(3)$ & 0.333 & -1.118 & 0.272 & -0.823 & -0.551 \\
\hline$(3,-1)$ & $\mathrm{N}(1)-\mathrm{C}(11)$ & 0.305 & -0.888 & 0.250 & -0.721 & -0.472 \\
\hline$(3,+1)$ & & 0.012 & 0.061 & 0.012 & -0.009 & 0.003 \\
\hline$(3,-1)$ & $\mathrm{N}(1)-\mathrm{H}(1)$ & 0.331 & -1.275 & 0.243 & -0.805 & -0.562 \\
\hline$(3,-1)$ & $\mathrm{N}(2)-\mathrm{C}(17)$ & 0.512 & -1.457 & 0.699 & -1.761 & -1.063 \\
\hline$(3,-1)$ & $\mathrm{C}(1)-\mathrm{C}(18)$ & 0.250 & -0.549 & 0.193 & -0.522 & -0.330 \\
\hline$(3,-1)$ & $\mathrm{C}(1)-\mathrm{H}(1 \mathrm{~B})$ & 0.281 & -0.804 & 0.213 & -0.627 & -0.414 \\
\hline$(3,-1)$ & $\mathrm{C}(1)-\mathrm{H}(1 \mathrm{~A})$ & 0.294 & -0.855 & 0.230 & -0.674 & -0.444 \\
\hline$(3,-1)$ & $\mathrm{C}(2)-\mathrm{C}(3)$ & 0.257 & -0.656 & 0.189 & -0.542 & -0.353 \\
\hline$(3,-1)$ & $\mathrm{C}(2)-\mathrm{C}(18)$ & 0.260 & -0.649 & 0.197 & -0.556 & -0.359 \\
\hline$(3,-1)$ & $C(4)-C(5)$ & 0.307 & -0.672 & 0.290 & -0.748 & -0.458 \\
\hline$(3,+1)$ & & 0.021 & 0.139 & 0.028 & -0.021 & 0.007 \\
\hline$(3,-1)$ & $\mathrm{C}(4)-\mathrm{C}(9)$ & 0.325 & -0.936 & 0.285 & -0.804 & -0.519 \\
\hline$(3,-1)$ & $C(5)-C(6)$ & 0.315 & -0.796 & 0.285 & -0.770 & -0.484 \\
\hline$(3,-1)$ & $\mathrm{C}(5)-\mathrm{H}(5)$ & 0.289 & -0.840 & 0.224 & -0.657 & -0.434 \\
\hline$(3,-1)$ & $C(6)-C(7)$ & 0.331 & -0.961 & 0.294 & -0.829 & -0.534 \\
\hline$(3,-1)$ & $\mathrm{C}(6)-\mathrm{H}(6)$ & 0.298 & -0.948 & 0.224 & -0.684 & -0.460 \\
\hline$(3,-1)$ & $C(7)-C(8)$ & 0.339 & -1.079 & 0.294 & -0.858 & -0.564 \\
\hline$(3,-1)$ & $\mathrm{C}(8)-\mathrm{C}(9)$ & 0.330 & -0.892 & 0.305 & -0.833 & -0.528 \\
\hline$(3,-1)$ & $\mathrm{C}(8)-\mathrm{H}(8)$ & 0.289 & -0.830 & 0.225 & -0.658 & -0.433 \\
\hline
\end{tabular}




$\begin{array}{lcccccc}(3,-1) & \mathrm{C}(9)-\mathrm{H}(9) & 0.285 & -0.818 & 0.219 & -0.642 & -0.423 \\ (3,-1) & \mathrm{C}(10)-\mathrm{C}(11) & 0.324 & -0.932 & 0.284 & -0.800 & -0.517 \\ (3,+1) & & 0.022 & 0.127 & 0.026 & -0.021 & 0.006 \\ (3,-1) & \mathrm{C}(10)-\mathrm{C}(15) & 0.320 & -0.889 & 0.281 & -0.785 & -0.504 \\ (3,-1) & \mathrm{C}(11)-\mathrm{C}(16) & 0.326 & -0.963 & 0.284 & -0.809 & -0.525 \\ (3,-1) & \mathrm{C}(11)-\mathrm{H}(11) & 0.283 & -0.801 & 0.217 & -0.634 & -0.417 \\ (3,-1) & \mathrm{C}(12)-\mathrm{C}(17) & 0.309 & -0.833 & 0.266 & -0.741 & -0.475 \\ (3,-1) & \mathrm{C}(12)-\mathrm{C}(16) & 0.285 & -0.844 & 0.214 & -0.638 & -0.425 \\ (3,-1) & \mathrm{C}(14)-\mathrm{C}(17) & 0.321 & -0.839 & 0.291 & -0.792 & -0.501 \\ (3,-1) & \mathrm{C}(13)-\mathrm{C}(17) & 0.292 & -0.800 & 0.235 & -0.670 & -0.435 \\ (3,-1) & \mathrm{C}(14)-\mathrm{C}(15) & 0.323 & -0.940 & 0.281 & -0.797 & -0.516 \\ (3,-1) & \mathrm{C}(14)-\mathrm{H}(14) & 0.291 & -0.906 & 0.216 & -0.659 & -0.443 \\ (3,-1) & \mathrm{C}(15)-\mathrm{H}(15) & 0.293 & -0.905 & 0.221 & -0.668 & -0.447 \\ (3,-1) & \mathrm{C}(18)-\mathrm{H}(18 \mathrm{~A}) & 0.272 & -0.615 & 0.225 & -0.604 & -0.379 \\ (3,-1) & \mathrm{C}(18)-\mathrm{H}(18 \mathrm{~B}) & 0.254 & -0.623 & 0.189 & -0.533 & -0.344 \\ (3,-1) & \mathrm{C}(18)-\mathrm{H}(18 \mathrm{C}) & 0.260 & -0.579 & 0.207 & -0.560 & -0.352\end{array}$


Table S2 The QTAIM integrated parameters for Bic.

\begin{tabular}{|c|c|c|c|c|c|}
\hline Atom & $\mathrm{Q}_{\exp }$ & $\mathrm{V}_{\mathrm{AIM}}$ & Atom & $\mathrm{Q}_{\mathrm{exp}}$ & $\mathrm{V}_{\text {AIM }}$ \\
\hline S1 & 1.65 & 8.21 & C12 & 0.32 & 9.70 \\
\hline F1 & -0.64 & 17.65 & C13 & 0.21 & 11.39 \\
\hline F2 & -0.81 & 17.22 & C14 & 0.29 & 11.02 \\
\hline F3 & -0.66 & 17.35 & C15 & 0.27 & 10.52 \\
\hline F4 & -0.75 & 16.42 & C16 & 1.64 & 3.54 \\
\hline N1 & -2.37 & 16.07 & C17 & 1.01 & 10.89 \\
\hline N2 & -2.30 & 18.31 & C18 & 0.11 & 9.00 \\
\hline 01 & -1.05 & 14.76 & H3 & 0.55 & 2.56 \\
\hline $\mathbf{O 2}$ & -1.21 & 18.13 & H1 & 0.47 & 2.80 \\
\hline $\mathbf{O 3}$ & -0.23 & 14.72 & H1B & -0.09 & 7.78 \\
\hline 04 & -0.11 & 22.00 & H1A & -0.08 & 6.91 \\
\hline C1 & 0.05 & 7.58 & H5 & -0.09 & 6.54 \\
\hline C2 & 0.76 & 5.06 & H6 & -0.11 & 9.20 \\
\hline $\mathrm{C3}$ & 1.59 & 4.82 & H8 & -0.07 & 6.87 \\
\hline $\mathrm{C4}$ & -0.05 & 11.37 & H9 & -0.03 & 8.79 \\
\hline C5 & 0.31 & 10.88 & H11 & -0.04 & 6.70 \\
\hline C6 & 0.13 & 11.25 & H14 & -0.10 & 8.73 \\
\hline C7 & 0.90 & 7.50 & H15 & -0.07 & 6.70 \\
\hline C8 & -0.14 & 11.88 & H18A & 0.00 & 6.49 \\
\hline C9 & 0.16 & 12.19 & H18B & -0.02 & 7.48 \\
\hline C10 & 0.45 & 9.97 & H18C & -0.01 & 8.01 \\
\hline C11 & 0.18 & 10.48 & & & \\
\hline
\end{tabular}


Table S3 Parameters of intermolecular contacts in Bic

\begin{tabular}{|c|c|c|c|c|c|c|}
\hline Atom1 & Atom2 & $\mathrm{R}, \AA$ & $\rho(r)$, a.u. & $\nabla^{2} \rho(\mathbf{r})$, a.u. & $\mathbf{V}(\mathbf{r})$, a.u. & $\mathrm{E}_{\text {bond }}, \mathrm{kJ} / \mathrm{mol}$ \\
\hline \multirow[t]{2}{*}{ F1 } & $\mathrm{F} 4$ & 3.061 & 0.003 & 0.017 & -0.002 & -2.4 \\
\hline & O3 & 3.275 & 0.003 & 0.015 & -0.002 & -2.1 \\
\hline \multirow[t]{2}{*}{$\mathrm{F} 2$} & H6 & 2.432 & 0.006 & 0.032 & -0.004 & -5.2 \\
\hline & F3 & 3.334 & 0.002 & 0.009 & -0.001 & -1.1 \\
\hline \multirow[t]{3}{*}{$\mathrm{F} 3$} & C6 & 3.311 & 0.003 & 0.011 & -0.001 & -1.7 \\
\hline & H1b & 2.628 & 0.003 & 0.015 & -0.002 & -2.1 \\
\hline & H9 & 3.247 & 0.001 & 0.005 & 0.000 & -0.6 \\
\hline \multirow[t]{3}{*}{$\mathrm{F} 4$} & $\mathrm{H} 1 \mathrm{~b}$ & 2.783 & 0.002 & 0.011 & -0.001 & -1.5 \\
\hline & $\mathrm{H} 18 \mathrm{c}$ & 3.061 & 0.003 & 0.014 & -0.002 & -2.0 \\
\hline & C13 & 3.285 & 0.004 & 0.016 & -0.002 & -2.6 \\
\hline $\mathrm{O} 1$ & H8 & 2.255 & 0.010 & 0.034 & -0.008 & -10.0 \\
\hline \multirow[t]{4}{*}{$\mathrm{O} 2$} & H5 & 2.479 & 0.006 & 0.025 & -0.003 & -4.1 \\
\hline & H11 & 2.402 & 0.006 & 0.030 & -0.004 & -4.7 \\
\hline & H8 & 2.953 & 0.003 & 0.012 & -0.001 & -1.8 \\
\hline & H9 & 2.871 & 0.003 & 0.014 & -0.002 & -2.1 \\
\hline \multirow[t]{3}{*}{ O4 } & H3 & 2.223 & 0.012 & 0.049 & -0.008 & -10.5 \\
\hline & $\mathrm{H} 18 \mathrm{a}$ & 2.58 & 0.006 & 0.025 & -0.003 & -4.3 \\
\hline & H14 & 2.554 & 0.004 & 0.022 & -0.002 & -3.1 \\
\hline \multirow[t]{5}{*}{ N2 } & H15 & 2.418 & 0.008 & 0.036 & -0.005 & -6.3 \\
\hline & $\mathrm{H} 18 \mathrm{a}$ & 2.665 & 0.008 & 0.027 & -0.004 & -5.4 \\
\hline & H1a & 2.981 & 0.009 & 0.031 & -0.005 & -6.0 \\
\hline & $\mathrm{H} 18 \mathrm{~b}$ & 2.962 & 0.004 & 0.015 & -0.002 & -2.3 \\
\hline & C11 & 3.416 & 0.004 & 0.014 & -0.002 & -2.5 \\
\hline C7 & C6 & 3.392 & 0.005 & 0.015 & -0.002 & -2.6 \\
\hline $\mathrm{C} 8$ & H11 & 3.031 & 0.004 & 0.013 & -0.002 & -2.3 \\
\hline \multirow[t]{2}{*}{ C15 } & C15 & 3.488 & 0.004 & 0.011 & -0.001 & -1.8 \\
\hline & $\mathrm{H} 18 \mathrm{a}$ & 3.129 & 0.004 & 0.013 & -0.002 & -2.2 \\
\hline H5 & $\mathrm{H} 8$ & 2.413 & 0.004 & 0.014 & -0.002 & -2.2 \\
\hline H9 & H9 & 2.853 & 0.002 & 0.007 & -0.001 & -0.9 \\
\hline H14 & $\mathrm{H} 18 \mathrm{~b}$ & 2.485 & 0.002 & 0.009 & -0.001 & -1.2 \\
\hline $\mathrm{H} 18 \mathrm{~b}$ & $\mathrm{H} 18 \mathrm{~b}$ & 2.992 & 0.005 & 0.019 & -0.002 & -3.0 \\
\hline
\end{tabular}

* Contacts are found and verified using WinXPRO program. The environment of $\mathbf{1}$ was generated as cluster and contacts inside this cluster were analyzed. 


\section{Bicalutamide in the binding pocket}

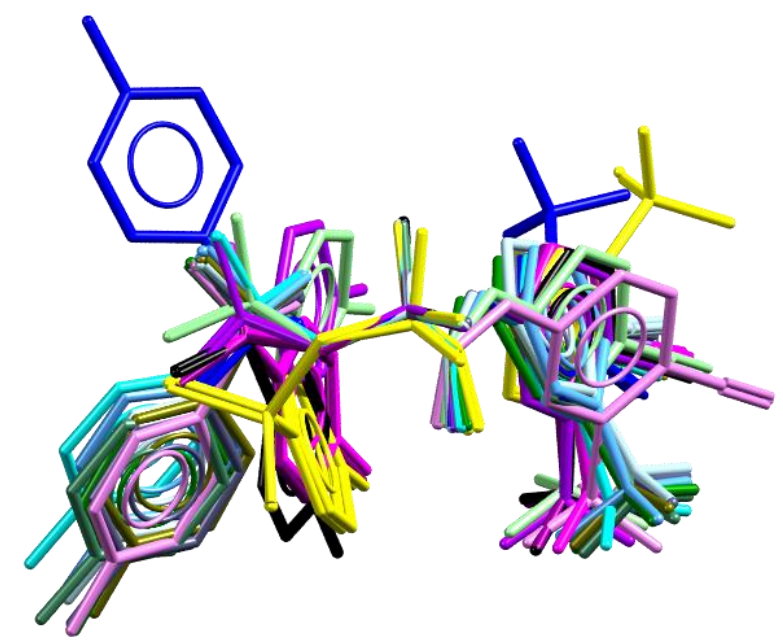

Figure S8 Molecular view of bicalutamide conformations in two polymorphs (monoclinic, blue; triclinic, black), four co-crystals (pink, magenta, violet, and light-bourdon) and ten complexes with macromolecule (yellow, light-blue and green colours). Hydrogen atoms are omitted. Superimposed atoms are the chiral carbon atoms and their four neighbours. 
Table S4 Table 4. Electrostatic interaction energy $\left(\mathrm{kJ} \mathrm{mol}^{-1}\right)$ between Bic and residues of LBP of the

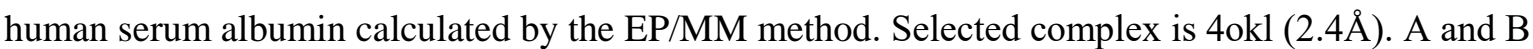
description indicate the chain name.

LEU

VAL

ARG

PRO

VAL

MET

ALA

THR

PHE

LEU

LYS

TYR

GLU

TYR

PHE

LEU

ARG

\section{human serum albumin}

4okl_A 4okl_B

$115 \quad-0.1 \quad 0.5$

$\begin{array}{lll}116 & 0.0 & 0.0\end{array}$

$\begin{array}{lll}117 & 5.4 & 5.2\end{array}$

$\begin{array}{lll}118 & 0.6 & 0.6\end{array}$

$\begin{array}{lll}122 & 0.0 & 0.0\end{array}$

$123 \quad-0.1 \quad 0.0$

$\begin{array}{lll}126 & -0.7 & -0.7\end{array}$

$\begin{array}{lll}133 & 0.0 & 0.0\end{array}$

$\begin{array}{lll}134 & 0.2 & 0.2\end{array}$

$\begin{array}{lll}135 & -0.2 & -0.2\end{array}$

$\begin{array}{lll}137 & -4.7 & -4.5\end{array}$

$\begin{array}{lll}138 & 0.3 & 0.3\end{array}$

$\begin{array}{lll}141 & 1.9 & 1.7\end{array}$

$\begin{array}{lll}161 & -0.2 & -0.2\end{array}$

$\begin{array}{lll}165 & 0.0 & 0.1\end{array}$

$182-0.2 \quad-0.1$

$186 \quad-1.3 \quad-1.3$

$\Sigma$

$\begin{array}{ll}0.9 & 1.4\end{array}$




\section{References}

[S1] Meindl, K; Herbst-Irmer, R.; Henn, J. (2010). Acta Crystallogr. A66, 362-371.

[S2] Meindl, K; Henn, J. (2008). Acta Crystallogr. A64, 404-418.

[S3] Hubschle, C. B.; Dittrich, B. (2011). J. Appl. Crystallogr. 44, 238-240.

[S4] Zhurova, E.A.; Zhurov, V.V.; Kumaradhas, P.; Cenedese, P.; Pinkerton, A.A.; J. Phys. Chem. B., 34, $120,8882-8891$

[S5] Zhurov, V. V.; Zhurova, E. A. \& Pinkerton, A. A. (2008). J. Appl. Crystallogr. 41, 340-349.

[S6] Henn, J. \& Meindl, K. (2014). Acta Crystallogr. A70, 248-256. 